\title{
Sunscreen Posts on Twitter in the United States and Canada, 2019: Content Analysis
}

Sajjad S Fazel ${ }^{1,2,3}$, PharmD, MPH; Emma K Quinn ${ }^{2,3,4}$, BSc; Chelsea A Ford-Sahibzada ${ }^{2,3}$, BSc; Steven Szarka ${ }^{3}$, PhD; Cheryl E Peters ${ }^{1,2,3}, \mathrm{PhD}$

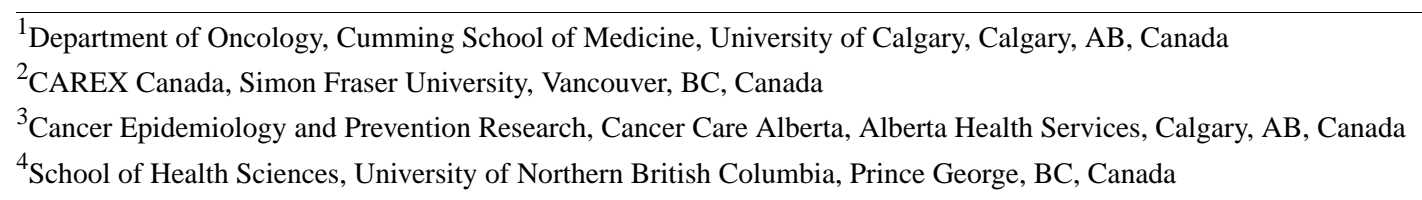

\section{Corresponding Author:}

Sajjad S Fazel, PharmD, MPH

Department of Oncology

Cumming School of Medicine

University of Calgary

3330 Hospital Dr NW

Calgary, $\mathrm{AB}, \mathrm{T} 2 \mathrm{~N} 4 \mathrm{~N} 1$

Canada

Email: sajjad.fazel@ucalgary.ca

(JMIR Dermatol 2021;4(2):e29723) doi: 10.2196/29723

\section{KEYWORDS}

sunscreen; skin cancer; Twitter; misinformation; prevention; skin; social media; health promotion; melanoma

Skin cancer is a growing burden in Canada and the United States. One effective prevention method is the use of sunscreen; however, low sunscreen use [1] coupled with the spread of misinformation online can hinder health promotion activities.

Health-related social media posts (including sunscreen) may shape risk-related behaviors of users, so it is important to understand the accuracy of such posts [2].

Twitter's Application Program Interface was used to search for tweets in English containing the word "sunscreen" posted in Canada and the United States (May 1 to August 31, 2019). We used thematic content analysis to elicit the accuracy, sentiment, and theme of the tweets.

Tweets containing verifiable information (that could be assessed as factual or not) were analyzed for accuracy and coded as either "accurate" or "inaccurate" based on current evidence. All tweets were coded for sentiment (positive or negative).
Themes were analyzed using an a priori list of codes based on our previous study [3] and inductively modified based on emergent themes. Differences were tested using the chi-square statistic or the Fisher exact test.

In total, 9176 tweets were collected; 167 retweets and 85 irrelevant tweets were excluded. The remaining 8924 tweets were analyzed for accuracy (where applicable), sentiment, and theme. The observed percentage agreement between the coders for sentiment and accuracy was $76 \%$. Only 395 tweets (4\% of the total) contained verifiable information and were analyzed for accuracy. Among these, 277 (70\%) were accurate and 118 $(30 \%)$ were inaccurate (Figure 1).

The most common themes were personal story $(n=5425,61 \%)$, tips and recommendations ( $\mathrm{n}=2591,28 \%)$, and advertisements $(n=457,5 \%)$. The top theme for accurate and inaccurate tweets was tips and recommendations $(\mathrm{n}=171,56 \%)$ and personal story $(n=90,62 \%)$, respectively. 
Figure 1. Comparison of sentiments between accurate, inaccurate, and unverifiable sunscreen tweets originating from Canada and United States between May 1, 2019 and August 31, 2019.

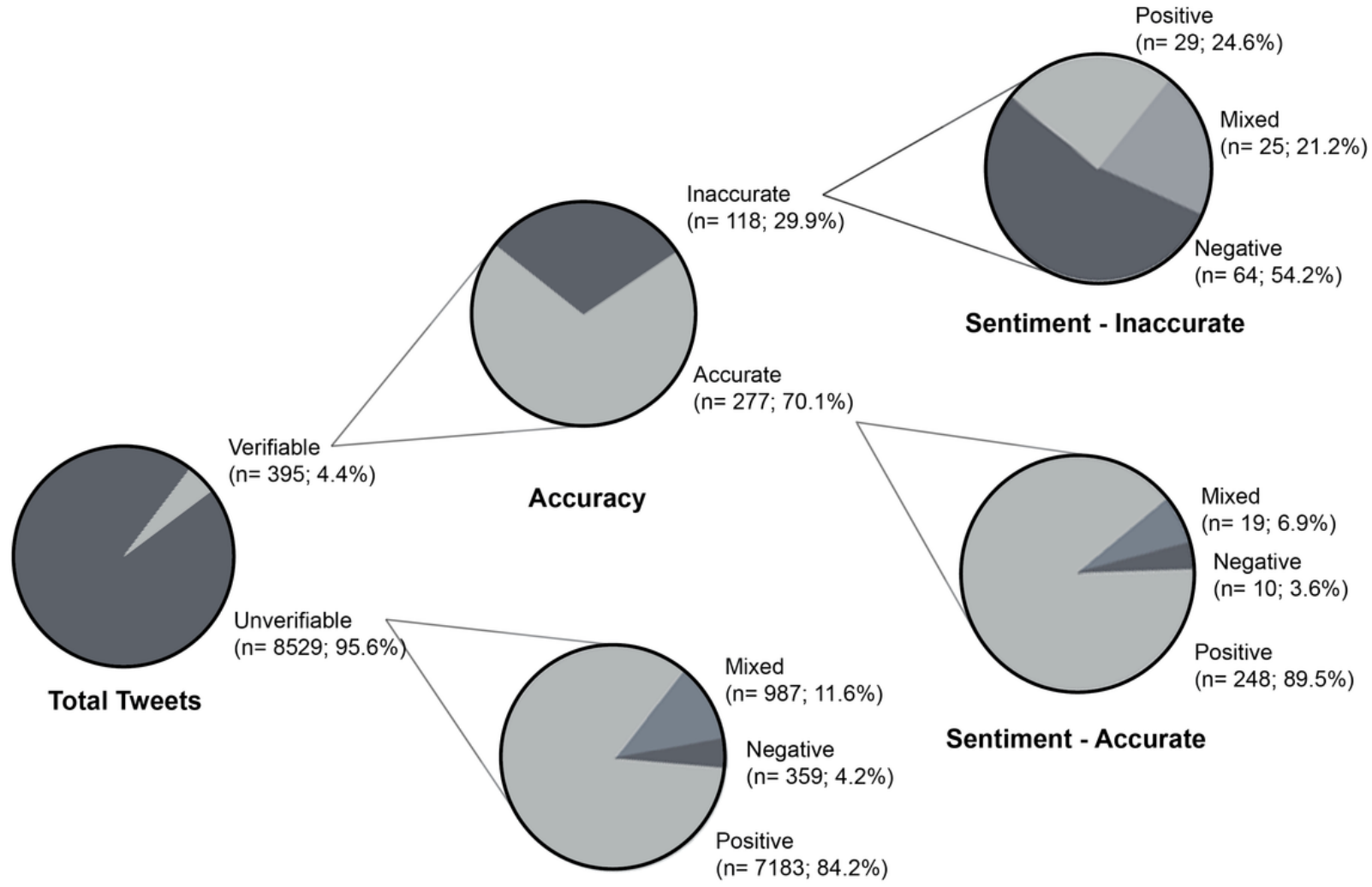

Sentiment - Unverifiable

The sentiment analysis found that $7460(84 \%)$ of tweets had a positive sentiment, $1031(11 \%)$ were mixed or neutral, and 433 (5\%) were negative. Among the accurate tweets, the majority had a positive sentiment toward sunscreen $(n=248,89 \%)$, while over half $(n=64,54 \%)$ of the inaccurate tweets had a negative sentiment. Interestingly, inaccurate tweets were more likely to have any engagement than accurate tweets (Table 1).

We found that most tweets were personal stories and not verifiable for accuracy. This suggests that misinformation about sunscreen may not be an important contributor to low sunscreen use, as also noted by Silva et al [4]. The sentiment analysis found that over $80 \%$ of all sunscreen tweets had a positive sentiment toward sunscreen use, which is similar to our previous study on sunscreen information in traditional media sources [3].

This study was limited to Twitter; further research on sunscreen misinformation using other social media platforms is recommended.

In conclusion, sunscreen misinformation was limited, but misinformation was more likely to have engagement from users. Organizations may have better success in promoting sunscreen use by producing tailored, engaging sunscreen and cancer prevention messages [5]. Furthermore, it may be beneficial for physicians and health organizations to share messages using lived experience, which may increase reach and engagement online. 
Table 1. Comparison of Twitter data between verifiable and unverifiable tweets: Canada and United States, 2019.

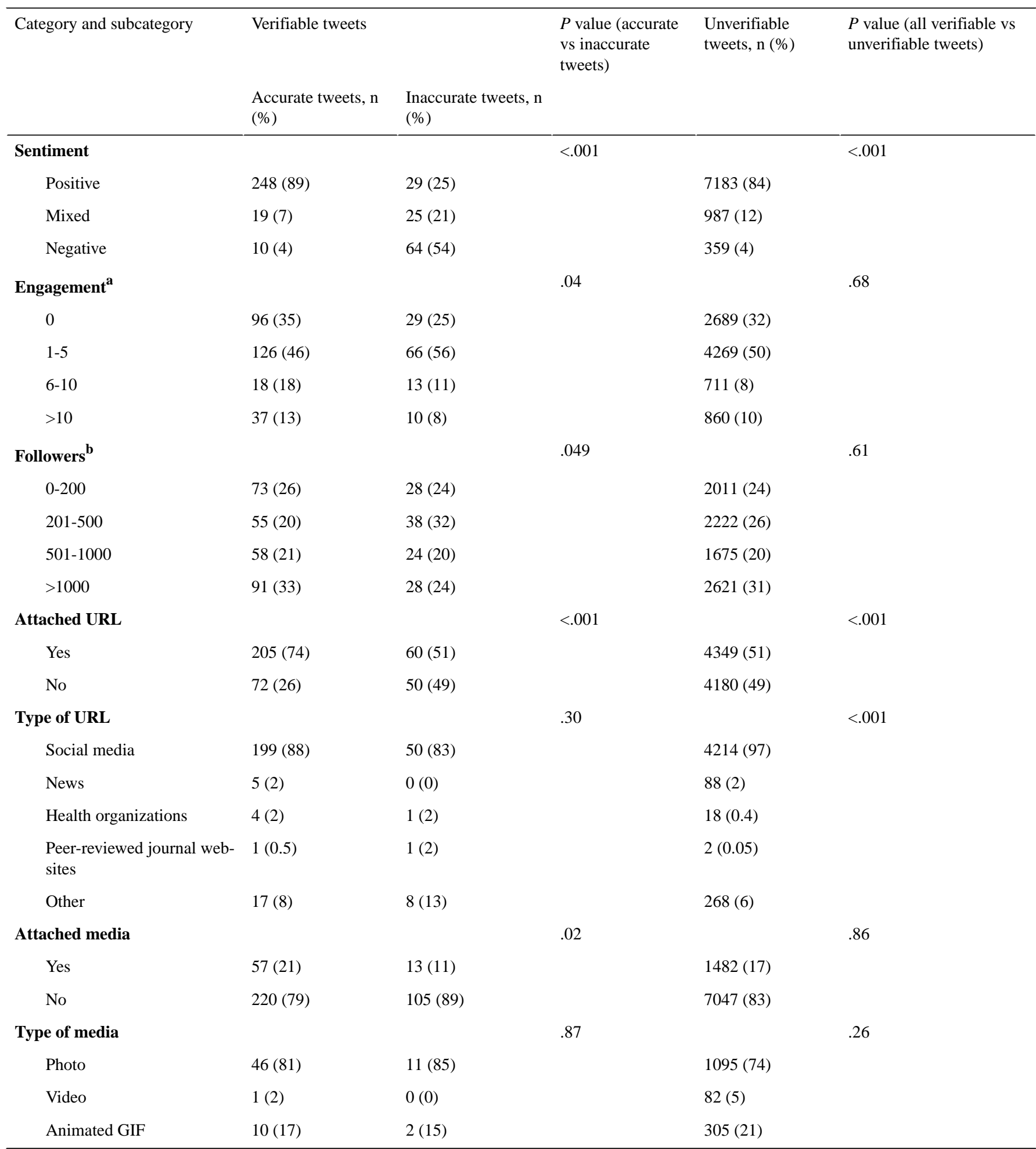

aEngagement was defined as the total number of "likes," "retweets," "quote tweets," and "replies" for each tweet.

${ }^{\mathrm{b}}$ Followers was defined as the number of individual Twitter accounts following the user.

\section{Acknowledgments}

This research received no external funding.

\section{Conflicts of Interest}

None declared. 


\section{References}

1. Pinault L, Fioletov V. Sun exposure, sun protection and sunburn among Canadian adults (Vol 28, Issue 5). Statistics Canada. 2017 May 17. URL: https://www150.statcan.gc.ca/n1/pub/82-003-x/2017005/article/14792-eng.htm [accessed 2021-07-07]

2. Macnamara J. Media content analysis: Its uses; benefits and best practice methodology. Asia Pacific Public Relations Journal 2010;6(1):1-34 [FREE Full text]

3. Quinn E, Ford Sahibzada C, Fazel S, McWhirter J, Peters C. Sunscreen and Associated Risk in the News: A Content Analysis of Canadian Newspapers (2009-2019). The Journal of Communication and Media Studies 2021;6(1):41-55. [doi: 10.18848/2470-9247/CGP/v06i01/41-55]

4. Vasconcelos Silva C, Jayasinghe D, Janda M. What Can Twitter Tell Us about Skin Cancer Communication and Prevention on Social Media? Dermatology 2020 Feb 25;236(2):81-89 [FREE Full text] [doi: 10.1159/000506458] [Medline: 32097934]

5. Caulfield T. Pseudoscience and COVID-19 — we've had enough already. Nature 2020 Apr 27. [doi: $\underline{10.1038 / \mathrm{d} 41586-020-01266-\mathrm{z}]}$

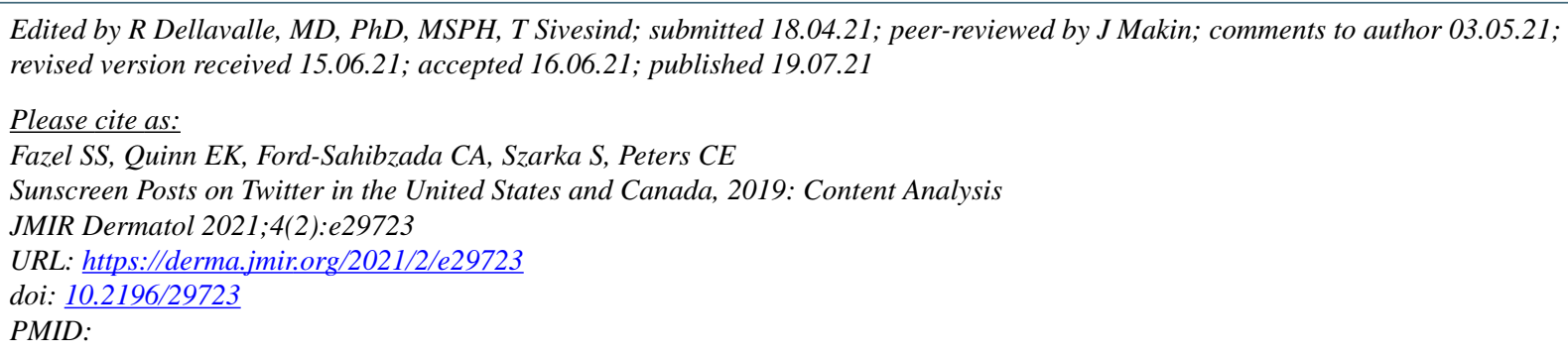

(CSajjad S Fazel, Emma K Quinn, Chelsea A Ford-Sahibzada, Steven Szarka, Cheryl E Peters. Originally published in JMIR Dermatology (http://derma.jmir.org), 19.07.2021. This is an open-access article distributed under the terms of the Creative Commons Attribution License (https://creativecommons.org/licenses/by/4.0/), which permits unrestricted use, distribution, and reproduction in any medium, provided the original work, first published in JMIR Dermatology Research, is properly cited. The complete bibliographic information, a link to the original publication on http://derma.jmir.org, as well as this copyright and license information must be included. 